\title{
A novel mutation of the calcium-sensing receptor gene in a German subject with familial hypocalciuric hypercalcemia and primary hyperparathyroidism
}

\author{
Marios Papadakis, Natalie Meurer, Theodora Margariti, Anke Meyer, \\ Norbert Weyerbrock, Cornelia Dotzenrath
}

Department of Endocrine Surgery, Helios Clinic, University Hospital Witten-Herdecke, Germany

\begin{abstract}
OBJECTIVE: The coexistence of familial hypocalciuric hypercalcemia (FHH) and primary hyperparathyroidism (PHPT) is extremely rare. Genetic evidence has demonstrated a causal relationship between $\mathrm{FHH}$ and the presence of inactivating mutations in the calcium-sensing receptor gene. METHOD: We herein report a 60 -year-old German patient who was referred for hypercalcemia and increased PTH levels found incidentally during normal routine blood tests. RESULTS: The patient underwent surgical exploration and the diagnosis of PHPT was histologically confirmed. One week later, the follow-up blood tests revealed recurrent hypercalcemia, and the possibility of FHH was reconsidered. Genetic analysis was performed and revealed a novel heterozygous CaSR single missense mutation (Arg551Gly) within the extracellular CaSR domain. CONCLUSION: We report a novel heterozygous missense inactivating mutation within the extracellular CaSR domain in a German subject with FHH and histologically proven PHPT.
\end{abstract}

Key words: Calcium-sensing receptor, Familial hypocalciuric hypercalcemia, Missense mutation, Primary hyperparathyroidism

\section{INTRODUCTION}

Familial hypocalciuric hypercalcemia $(\mathrm{FHH})$ is an autosomal dominantly inherited disorder of calcium metabolism. Its main feature is mild to moderate hypercalcemia, mostly persistent and non-progressive.

\footnotetext{
Address for correspondence:

Marios Papadakis, M.D., Ph.D.; Helios Clinic, University Hospital Witten-Herdecke, Department of Plastic and Hand Surgery, Heusnerstr. 40, 42283 Wuppertal, Germany;

E-mail: marios_papadakis@yahoo.gr

Received: 20-11-2016, Accepted: 16-12-2016
}

Parathyroid hormone levels range from normal to mildly elevated. Unlike primary hyperparathyroidism (PHPT), FHH is characterized by hypocalciuria. PHPT requires surgical treatment, i.e. parathyroidectomy, which is contraindicated for FHH. However, not infrequently, both pathologies are present. Genetic evidence has identified a causal relationship between FHH and the presence of inactivating mutations in the calcium-sensing receptor (CaSR) gene. The CaSR gene encodes the calcium sensing receptor, a plasmamembrane calcium-binding G-protein coupled receptor, 
which is predominantly expressed in the parathyroid glands, the thyroid $\mathrm{C}$ cells and the kidneys. CaSR consists of three domains: an extracellular domain, which binds extracellular calcium, a seven transmembrane domain and an intracellular signal transduction domain, responsible for $\mathrm{Ca}^{2+}$-mediated activation of $\mathrm{G}$ proteins. The majority of FHH-related mutations are located in the extracellular CaSR domain, but mutations of the region of the gene encoding the signal-transducing domain have also been reported. ${ }^{1}$

We herein report a German patient with FHH and histologically proven PHPT, carrying a novel CaSR gene mutation. The patient was informed that data concerning the case would be submitted for publication, to which he gave his consent.

\section{CASE REPORT}

A 61-year-old male patient was referred for hypercalcemia found incidentally during normal routine blood tests. He had no personal or family history of nephrolithiasis, osteopathy, depression or gastrointestinal symptoms. The initial blood investigation revealed normal serum albumin levels (43 g/l, normal range 34-48 g/l), albumin corrected calcium levels of $3.1 \mathrm{mmol} / 1$ (normal: $2.2-2.6 \mathrm{mmol} / \mathrm{l}$ ), decreased 25-OH-Vitamin D3 levels $(10.7 \mathrm{ng} / \mathrm{ml}$, normal range $>20 \mathrm{ng} / \mathrm{ml})$, normal creatinine levels $(0.88 \mathrm{mg} / \mathrm{dl}$, normal range $<1.1 \mathrm{mg} / \mathrm{dl}$ ), decreased serum phosphate levels ( $1 \mathrm{mmol} / 1$; normal $1.2-3.2 \mathrm{mmol} / \mathrm{l}$ ) and elevated serum PTH levels (137 pg/ml; normal 12-65 pg/ml). Alkaline phosphatase was normal $(77 \mathrm{U} / 1$, normal range 35-105 U/1). The patient's $24 \mathrm{~h}$ urinary calcium excretion was within the normal range and the calcium/creatinine clearance ratio was clearly consistent with PHPT at 0.14 . Both neck ultrasonography and Sestamibi scan were performed and were negative for parathyroid adenoma.

The patient underwent surgical exploration, which disclosed a slightly enlarged right upper parathyroid gland. The gland was removed and its histological examination revealed hyperplastic nodular parathyroid tissue consistent with parathyroid adenoma. The other parathyroid glands were found to be normal and left in situ. Intraoperative PTH levels drawn from the right and the left jugular vein decreased from $722 \mathrm{pg} / \mathrm{ml}$ to $81 \mathrm{pg} / \mathrm{ml}$ and from $233 \mathrm{pg} / \mathrm{ml}$ to $115 \mathrm{pg} /$ $\mathrm{ml}$, respectively, at $10 \mathrm{~min}$ post resection. One hour post surgery, peripheral parathormone level dropped to $35 \mathrm{pg} / \mathrm{ml}$. Serum calcium levels reached $2.5 \mathrm{mmol} / 1$ on the third postoperative day. However, one week later, the follow-up blood tests revealed recurrent hypercalcemia (2.9 mmol/1) and elevated PTH levels of $58 \mathrm{pg} / \mathrm{ml}$. The subsequent MRI and Sestamibi scan did not demonstrate any parathyroid adenoma and the possibility of FHH was reconsidered.

Genetic analysis was performed and identified a novel heterozygous CaSR single missense mutation (Arg551Gly) within the extracellular CaSR domain. The mutation was caused by a transversion of A to $\mathrm{G}$ detected at nucleotide position 1651 in exon 6 changing codon 551 (AGG $\rightarrow$ GGG). Further family screening was not performed, as the patient had no surviving relatives.

\section{DISCUSSION}

The coexistence of PHPT and FHH has been reported, though it is extremely rare. Interestingly, while mild parathyroid hyperplasia can occur in FHH patients, it usually concerns a lipohyperplasia characterized by simultaneous hyperplasia of fat and parathyroid principal cells. ${ }^{2}$ True parathyroid hyperplasia, as in our case, has also been reported, but it is not clear whether it is related to CaSR gene mutations or not. However, experimental model findings exist that support this relationship. ${ }^{2}$

Accurate diagnosis is important, as PHPT responds well to surgical treatment and FHH requires no treatment. The differential diagnosis is based on the calcium to creatinine $(\mathrm{Ca} / \mathrm{Cr})$ clearance ratio $(\mathrm{CCR})$. A CCR of less than 0.01 is typical of $\mathrm{FHH}$, whereas a CCR greater than 0.02 is suggestive of PHPT. However, this equation does not take into account ionized calcium and is, moreover, characterized by low specificity. Almost $1 / 3$ of patients with PHPT are shown to have a CCR suggestive of FHH, while another $1 / 3$ have a CCR of less than $0.02 .{ }^{3}$ In order to minimize such overlapping, some authors recommend the use of fasting urinary calcium excretion $(\mathrm{CaE})$ instead of CCR. ${ }^{3}$ Christensen et al report the gene analysis as having a sensitivity of $98 \%$ for FHH when applied in all hypercalcemic patients with a CCR of less than 0.02. ${ }^{1}$ In our case, the CCR was found to be 0.14 , a 
value which, taking into account the elevated serum PTH levels, was ascribed to PHPT, so that the $\mathrm{CaE}$ was not deemed necessary, this decision being based on the described intra-individual variation of renal calcium excretion. ${ }^{1}$ Both serum PTH and serum calcium levels reached the normal range directly after surgery, although the latter was close to the upper limit of normal values $(2.58 \mathrm{mmol} / \mathrm{l})$. The first week follow-up showed values that made the diagnosis of FHH likely.

FHH is associated with inactivating mutations in the CaSR gene located on chromosome 3q21-24. However, other loci (19p13.3 and 19q13) have also been reported. ${ }^{4}$ To date, more than $200 \mathrm{CaSR}$ FHH-related mutations have been identified worldwide (online database available at http://www.casrdb.mcgill.ca). ${ }^{5}$ Most mutations are missense and nonsense mutations, but deletions, insertions and splicing mutations have also been documented. The majority are localized in exons 3 and 4. Mutations located outside the coding exons 2-7 can result in negative CaSR gene tests in FHH patients. ${ }^{1}$

While heterozygous inactivating mutations cause $\mathrm{FHH}$, homozygous inactivating mutations are associated with neonatal severe hyperparathyroidism (NSHPT). The R551G mutation which we described has not, to the best of our knowledge, been previously reported. A thorough literature survey revealed another inactivating mutation in codon 551. The mutation predicted a substitution of arginine to lysine, not to glycine as in our case, and was associated with NSHPT. ${ }^{6}$ Inactivating missense mutations in codons 549 (G549R), ${ }^{7} 550$ (T550I), ${ }^{2} 553$ (G553R), ${ }^{8,9} 555$ $(\mathrm{I} 555 \mathrm{~V})^{8}$ and $557(\mathrm{G} 557 \mathrm{E})^{4}$ have been reported in FHH subjects and families. Like other FHH-related mutations in exon 6, the R551G affects the extracellular CaSR domain, resulting in CaSR function loss and therefore abnormal calcium sensing. Unfortunately, the R551G mutation could not be further established as no family members were available for genetic testing.

In conclusion, we report a novel heterozygous missense inactivating mutation of the CaSR in a German subject with FHH and histologically proven PHPT.

\section{REFERENCES}

1. Christensen SE, Nissen PH, Vestergaard P, Mosekilde L, 2011 Familial hypocalciuric hypercalcaemia: a review. Curr Opin Endocrinol Diabetes Obes 18: 359-370.

2. Al-Salameh A, Cetani F, Pardi E, et al, 2011 A novel mutation in the calcium-sensing receptor in a French family with familial hypocalciuric hypercalcaemia. Eur J Endocrinol 165: 359-363.

3. Glendenning P, 2003 Summary statement from a workshop on asymptomatic primary hyperparathyroidism: a perspective for the 21 st century. Clin Biochem Rev 24: 27-30.

4. Nakayama T, Minato M, Nakagawa M, et al, 2001 A novel mutation in $\mathrm{Ca} 2+$-sensing receptor gene in familial hypocalciuric hypercalcemia. Endocr 15: 277-282.

5. Zapanti E, Polonifi A, Kokkinos M, et al, 2015 A novel mutation of the calcium-sensing receptor gene in a Greek family from Nisyros. Hormones (Athens) 14: 321-325.

6. Tõke J, Czirják G, Patócs A, et al, 2007 Neonatal severe hyperparathyroidism associated with a novel de novo heterozygous $\mathrm{R} 551 \mathrm{~K}$ inactivating mutation and a heterozygous A986S polymorphism of the calcium-sensing receptor gene. Clin Endocrinol (Oxf) 67: 385-392.

7. D’Souza-Li L, Yang B, Canaff L, et al, 2002 Identification and functional characterization of novel calciumsensing receptor mutations in familial hypocalciuric hypercalcemia and autosomal dominant hypocalcemia. J Clin Endocrinol Metab 87: 1309-1318.

8. Nissen PH, Christensen SE, Heickendorff L, Brixen K, Mosekilde L, 2007 Molecular genetic analysis of the calcium sensing receptor gene in patients clinically suspected to have familial hypocalciuric hypercalcemia: phenotypic variation and mutation spectrum in a Danish population. J Clin Endocrinol Metab 92: 4373-4379.

9. Schwarz P, Larsen NE, Lønborg Friis IM, Lillquist K, Brown EM, Gammeltoft S, 2000 Familial hypocalciuric hypercalcemia and neonatal severe hyperparathyroidism associated with mutations in the human $\mathrm{Ca} 2+$-sensing receptor gene in three Danish families. Scand J Clin Lab Invest 60: 221-227. 